\title{
Standing-wave spectrometer
}

Helmut Stiebig, Dietmar Knipp, and Eerke Bunte

Citation: Appl. Phys. Lett. 88, 083509 (2006);

View online: https://doi.org/10.1063/1.2179610

View Table of Contents: http://aip.scitation.org/toc/apl/88/8

Published by the American Institute of Physics

\section{Articles you may be interested in}

The Spectrometer

The Physics Teacher 50, 152 (2012); 10.1119/1.3685111

Standing-wave interferometer

Applied Physics Letters 83, 12 (2003); 10.1063/1.1590732

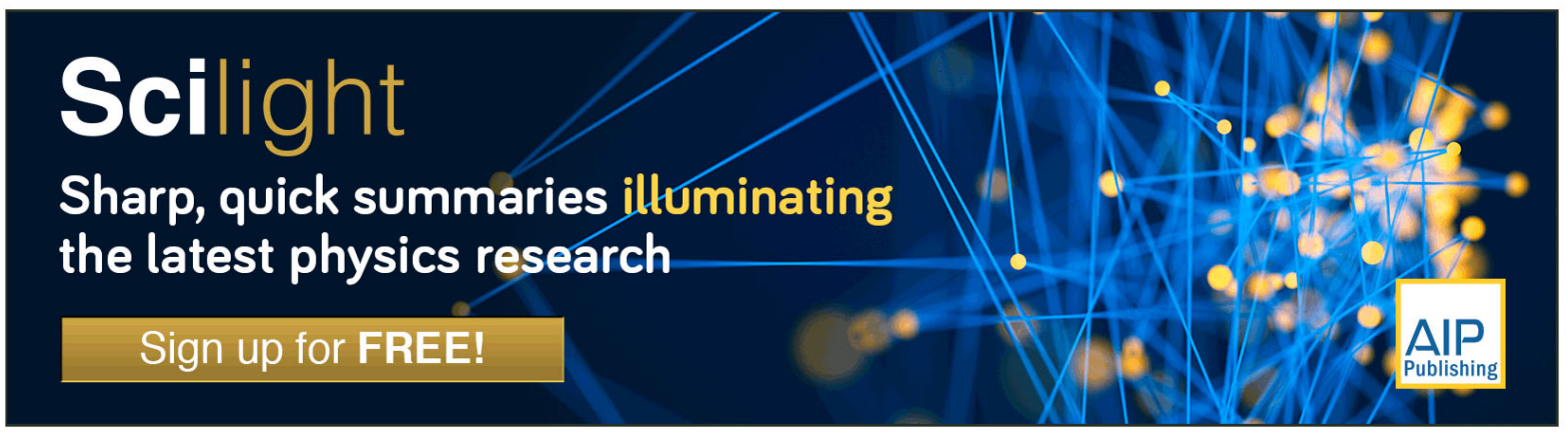




\title{
Standing-wave spectrometer
}

\author{
Helmut Stiebig \\ Institute of Photovoltaics, Research Center Jülich, 52425 Jülich, Germany \\ Dietmar Knipp ${ }^{\text {a) }}$ \\ Department of Engineering and Science, International University Bremen, 28759 Bremen, Germany
}

Eerke Bunte

Institute of Photovoltaics, Research Center Jülich, 52425 Jülich, Germany

(Received 14 June 2005; accepted 17 January 2006; published online 24 February 2006)

\begin{abstract}
A standing-wave sensor was developed which facilitates the miniaturization of Fourier spectrometers down to the micrometer scale. The spectrometer concept is based on sampling a standing wave by an ultrathin and partially transmissive sensor. The active region of the sensor has a thickness of $30 \mathrm{~nm}-40 \mathrm{~nm}$. The standing wave is created in front of a tunable mirror. Varying the position of the mirror results in a phase shift of the standing wave, a modulation of the intensity profile within the ultrathin sensor, and thus, in a modulation of the photocurrent. The spectral information of the incoming light can be determined by the Fourier transform of the sensor signal. The operation principle of the spectrometer is described and the influence of the device design on the spectral resolution of the spectrometer is discussed. Due to the simple linear setup of the sensor and the tunable mirror, the realization of one- and two-dimensional spectrometer arrays is feasible. (C) 2006 American Institute of Physics. [DOI: 10.1063/1.2179610]
\end{abstract}

Research on microelectromechanical systems and integrated photonic technologies has lead to the development of several concepts and devices in the area of optical metrology. Very often the operating principle of the devices is based on the interference of optical waves. ${ }^{1-6}$ In almost all cases, the detected interference pattern is created by the superposition of waves propagating in the same direction. Typical examples of such devices are Michelson Interferometers and Fourier spectrometers. ${ }^{2}$ In recent years, an alternative approach has been developed, which benefits from the superposition of waves propagating in opposite directions. ${ }^{3-6}$ The working principle of such devices is based on sampling a standing wave created in front of a mirror or a highly reflective object. ${ }^{3-6}$ The intensity profile of the standing is sensed by a partially transmissive optical sensor. So far, this approach has been used to realize standing-wave interferometers ${ }^{3,5}$ or Fourier spectrometers that operate in the near-infrared part of the optical spectrum. ${ }^{4}$

In this letter, we will present an alternative approach to realize a standing-wave Fourier spectrometer that operates in the visible part of the optical spectrum. Sampling a standing wave by a semiconductor device requires a highly transmissive and very thin sensor. Otherwise, no light is transmitted through the sensor and no standing wave is created. On the other hand, sufficient light has to be absorbed to generate an electrical signal. The active region of the sensor has to be much thinner than the wavelength of the light, so that the overall photocurrent follows the intensity pattern of the standing wave. Here, we will discuss the influence of the partially transmissive sensor on the spectral resolution of the spectrometer.

Amorphous silicon was used for the fabrication of the ultrathin diodes. The thin films were deposited in a multichamber plasma enhanced chemical vapor deposition system

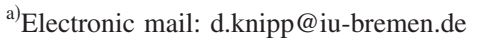

at $210{ }^{\circ} \mathrm{C}$ on glass substrates coated with transparent conductive oxide (TCO) layers. Deposition gases-silane, methane, and hydrogen-were used to prepare the ultrathin $n-i-p$ diodes. A small amount of carbon was alloyed to the silicon to increase the band gap $\left(E_{g}=2.0 \mathrm{eV}\right)$ and to decrease the refractive index of the material slightly. The intrinsic layer of the diode has a thickness between 30 and $40 \mathrm{~nm}$. After depositing the amorphous silicon $n-i-p$ diode, the second TCO-layer was prepared. The TCO layers were realized by magnetron sputtered $\mathrm{ZnO}{ }^{5}$ The devices were patterned using photolithography and reactive ion etching. A detailed description of the preparation conditions and the materials properties of the amorphous silicon diodes and the transparent oxide layers are given in Refs. 7 and 8.

The operating principle of the sensor is depicted in Fig. 1. The sensor consists of an ultrathin diode on a glass substrate, which is illustrated in Fig. 1 by a simple absorption layer with a refractive index of $n_{i}$ and a thickness of $d_{i}$. The reflection of the incoming intensity $l_{0}$ leads to the creation of a standing wave $\left(l_{s}\right)$ in front of the mirror, which changes its intensity from 0 to $4 \cdot l_{0}$ depending on the spatial position. Due to the modulation of the mirror along the $x$ axis, the

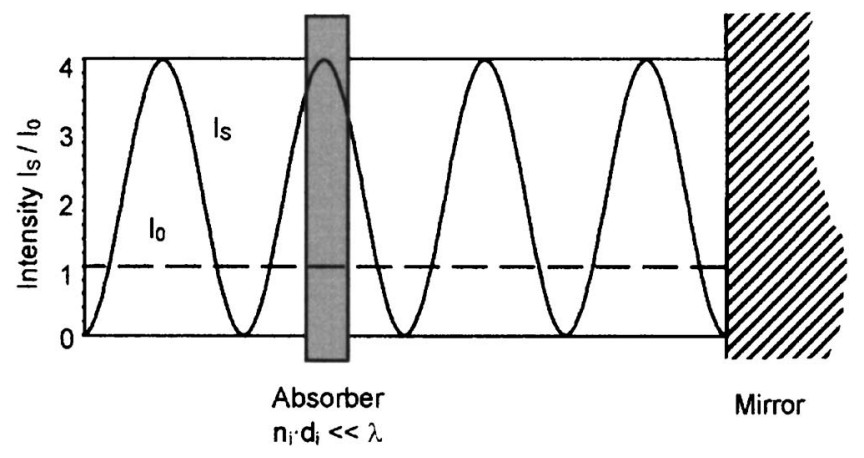

FIG. 1. Schematic setup of a standing-wave spectrometer consisting of a partially transmissive optoelectronic active absorber and a mirror. 


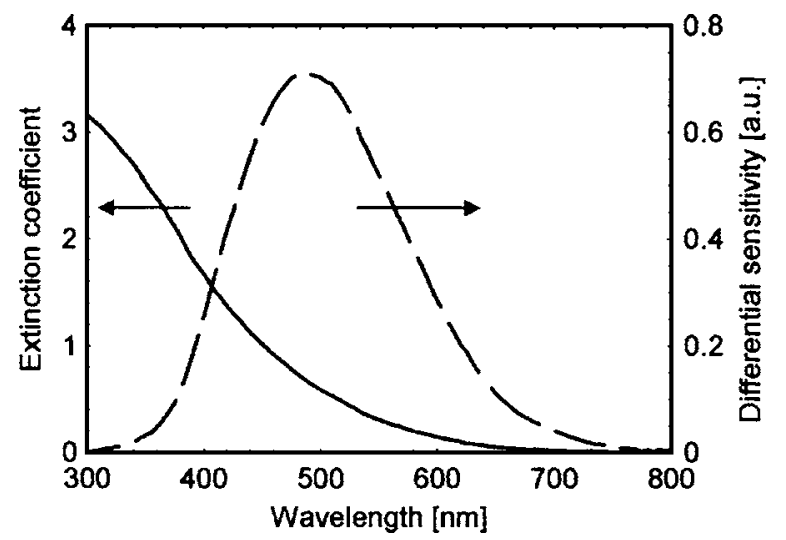

FIG. 2. Extinction coefficient of amorphous silicon and calculated differential sensitivity of an ultrathin diode. The thickness of the absorber region of the ultrathin amorphous silicon diode was assumed to be $35 \mathrm{~nm}$.

intensity profile of the standing wave shifts through the transmissive sensor leading to the modulation of the photocurrent. The wavelength of the standing wave corresponds to one-half of the wavelength of the incoming light. Figure 1 illustrates the ideal case, where no light is reflected or absorbed by the sensor. In the nonideal case, the light intensity reflected by the mirror is always smaller than the incoming light intensity due to the absorption of the incoming light and reflection losses at the interfaces of the detector and the mirror. Consequently, the modulation of the intensity is reduced $\left(0<l_{S}<4 \cdot l_{0}\right)$. To identify an optimized device design of the ultrathin sensor, we tried to maximize the differential sensitivity instead of maximizing the overall sensitivity of the sensor. We defined the differential sensitivity to be the alternating component of the photocurrent when the mirror is shifted, whereas the total sensitivity corresponds to the differential sensitivity plus the phase shift independent intensity (direct component of the sensitivity). The calculated differential sensitivity for an ultrathin diode with an absorber thickness of $35 \mathrm{~nm}$ is shown in Fig. 2. The differential sensitivity was calculated by considering the complex refractive index of amorphous silicon. The influences of reflections at the interfaces of the sensor on the differential spectral sensitivity were ignored in the calculation. The variation of the differential spectral sensitivity is caused by the strong wavelength dependent absorption of amorphous silicon (see extinction coefficient in Fig. 2).

For short wavelengths, the differential sensitivity of the sensor is very low as the extinction coefficient is very high. Therefore, only a small fraction of the incoming light is transmitted through the sensor, which contributes to the formation of a standing wave. For the visible part of the optical spectrum, the differential sensitivity of the sensor is high, since a large fraction of the light is transmitted through the sensor and a standing wave is formed in front of the mirror. For longer wavelengths, almost all of the light is transmitted through the partially transmissive sensor as the extinction coefficient of amorphous silicon is reduced. Due to the low extinction coefficient, the differential sensitivity drops for longer wavelengths.

Besides the thickness of the active region of the sensor, several other aspects have to be considered while designing the sensor structure. Optical simulations indicate that in particular the TCO layers have a strong influence on the device performance. Fabry-Perot oscillations are observed if the
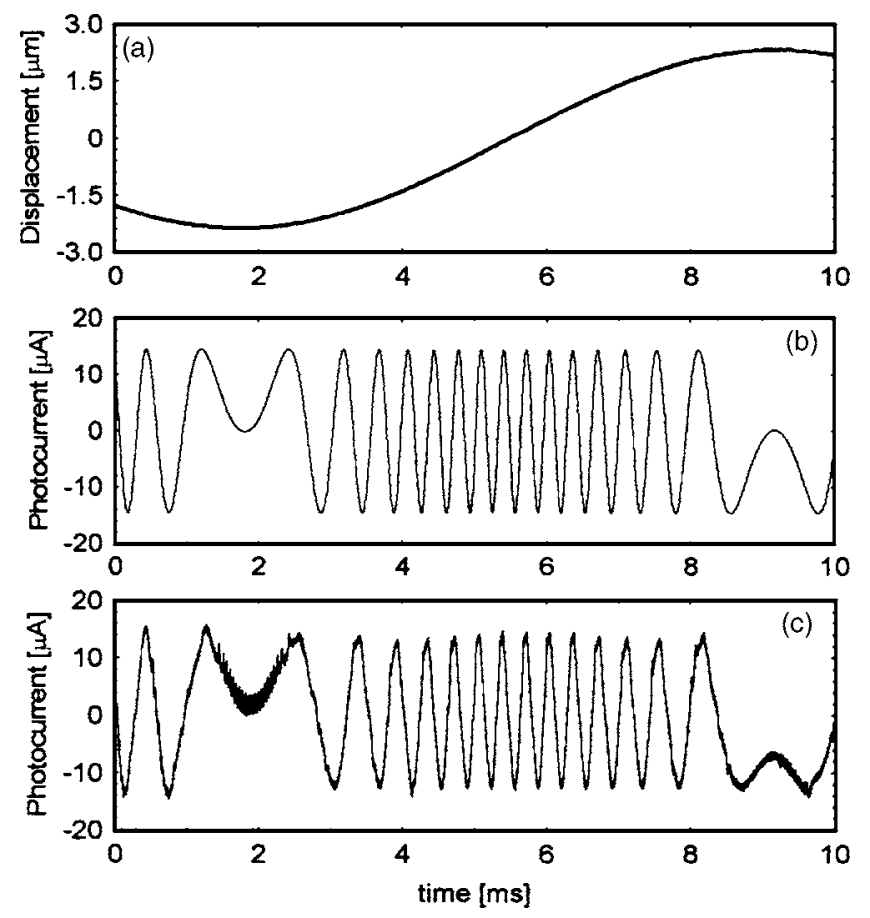

FIG. 3. Simulated and measured photocurrent of the standing-wave spectrometer upon modulation of the mirror by a sine function (a). The mirror was modulated by $4.68 \mu \mathrm{m}$ at a frequency of $67 \mathrm{~Hz}$. A HeNe laser source $(633 \mathrm{~nm})$ was used for the measurement of the alternating photocurrent in (c). Figure (b) shows the expected alternating photocurrent of the partially transmissive sensor.

TCO layers' thickness corresponds to an even multiple of $\lambda /\left(4 n_{\mathrm{TCO}}\right)$ where $n_{\mathrm{TCO}}$ is the refractive index of the transparent oxide layers. The oscillations lead to a nonlinear response of the sensor. A Fabry-Perot resonator is formed between the mirror and the detector. The Fabry-Perot oscillations can be minimized by using TCO layers, which have a thickness of odd multiples of $\lambda /\left(4 n_{\mathrm{TCO}}\right)$. Under such conditions, the TCO layer acts as an antireflection coating and the influence of the sensor structure on the propagation of the waves is minimized.

The measured photocurrent of the standing-wave spectrometer is given in Fig. 3. A HeNe laser emitting light at a wavelength of $633 \mathrm{~nm}$ was used to test the system, which consists of the ultrathin sensor and a piezo-actuated mirror. In Fig. 3(a), the displacement of the piezo-actuated mirror is given. The displacement of the mirror was measured by a capacitive sensor coupled to the actuator. The mirror was displaced by $4.68 \mu \mathrm{m}$ at a frequency of $67 \mathrm{~Hz}$. The alternating component of the photocurrent as a function of the mirror displacement is shown in Figs. 3(b) and 3(c). It can be clearly seen that the measured signal in Fig. 3(c) follows the modulating light intensity caused by constructive and destructive interference of the laser light. At $\lambda=633 \mathrm{~nm}$, the sensor has a transmission of $70 \%$ and a differential spectral sensitivity of $10 \mathrm{~mA} / \mathrm{W}-15 \mathrm{~mA} / \mathrm{W}$. A good agreement is observed between the calculated [Fig. 3(b)] and the measured [Fig. 3(c)] differential spectral sensitivity. The measured alternating component of the photocurrent follows a sine curve, which confirms that Fabry-Perot oscillations have only a minor influence on the measured photocurrent.

In the next step, we studied the influence of the mirror displacement on the spectral resolution of the spectrometer. 

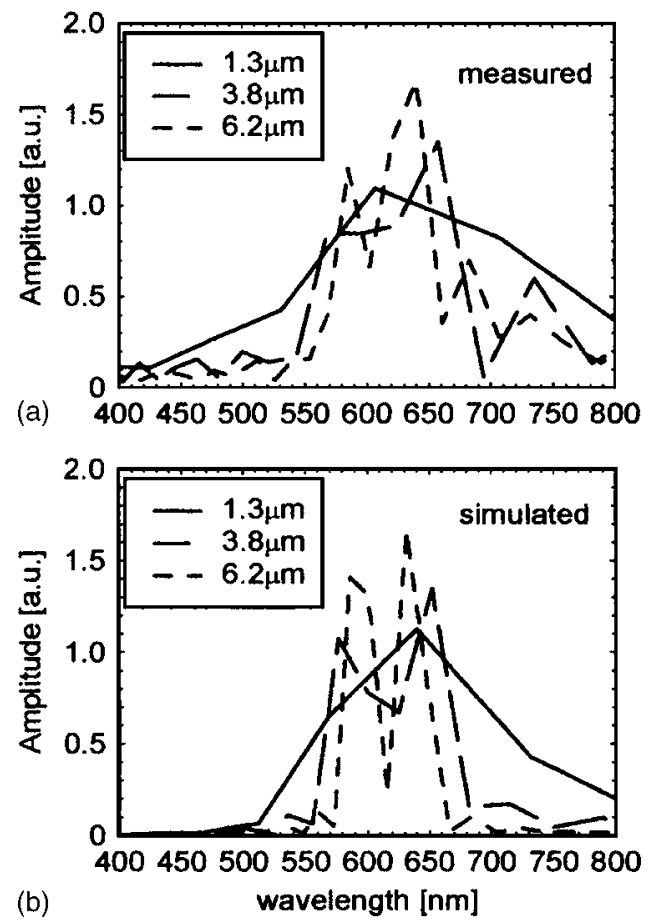

FIG. 4. Measured (a) and simulated (b) spectrum of two laser sources emitting at $594 \mathrm{~nm}$ and $633 \mathrm{~nm}$. The data were taken for different scan lengths of the spectrometer.

The spectral resolution of a spectrometer is, in general, given by

$$
\mathrm{FWHM}=\Delta \lambda=\frac{\lambda^{2}}{2 \cdot \Delta x},
$$

where $\lambda$ is the wavelength and $\Delta x$ is the scan length (displacement) of the mirror. In order to test the spectral resolution of the spectrometer, we used two laser sources which emit light at $594 \mathrm{~nm}$ and $633 \mathrm{~nm}$. The Fourier transform of the photocurrent spectra for different displacements is shown in Fig. 4(a). For a displacement of $1.3 \mu \mathrm{m}$, the two laser lines cannot be distinguished. Under these conditions, the spectral resolution of the spectrometer is $140 \mathrm{~nm}$, which is not sufficient to separate the two laser sources. With increasing displacement, the resolution of the spectrometer increases. The mirror was displaced up to $6.2 \mu \mathrm{m}$ so that the spectral resolution is increased to $30 \mathrm{~nm}$. Under such conditions, the two laser lines can be clearly distinguished. In Fig. 4(b), we simulated the spectral resolution of the spectrometer for different scan lengths assuming ideal conditions. A comparison of Figs. 4(a) and 4(b) indicates a good agreement between the measured data and the expected signals. In order to separate the two laser lines at $594 \mathrm{~nm}$ and $633 \mathrm{~nm}$, a displacement of $4.7 \mu \mathrm{m}$ is necessary. The measurements in Fig. 4(a) clearly confirm the theoretically expected spectral resolution.

In general, the partially transmissive sensor is optimized for a specific wavelength. As a consequence, the sensor is slightly mismatched for other wavelengths. This mismatch leads to: (i) A distortion of the wave propagation within the sensor and (ii) a wavelength dependent spectral sensitivity. These limitations can only be reduced but not prevented. Therefore, the spectrometer is of particular interest for applications, where the sensor signal is correlated to a reference signal, rather than being used as absolute measurement. Despite these limitations the spectrometer is able to fill the gap between standard cameras with three spectral channels for red, green, and blue and high-resolution grating spectrometers.

In terms of applications, it can be expected that the spectral resolution of the spectrometer is limited by the coherence length of the incoming light rather than the scan length of the tunable mirror. In order to minimize the influence of the coherence length on the spectral resolution, the tunable mirror has to be as close as possible to the partially transmissive sensor. In the ideal case, the partially transmissive sensor and the tunable mirror have to be integrated on a single substrate.

As the spectrometer consists only of the partially transmissive sensor in combination with an actuator, the number of required components is minimized. Furthermore, the linear setup of the sensor and the actuator allows for the integration of spectrometers. As these devices do not require any kind of beam splitter, several of these spectrometers can be densely packed on a chip. Further experiments are under way to realize such spectrometer arrays.

In summary, the concept of a Fourier spectrometer based on sampling a standing wave was presented. The sensor consists of a partially transmissive sensor in combination with a tunable mirror. Ultrathin amorphous silicon diodes prepared on glass substrates were used as partially transmissive sensors. The device is filling the gap between solid-state camera technology with only three-color channels but high spatial resolution on one hand, and precision spectrometers with high spectral resolution and no spatial resolution on the other hand.

Two of the authors (H. S. and E. B.) gratefully acknowledge the financial support from the "Deutsche Forschungsgemeinschaft".

${ }^{1}$ E. B. Cooper, E. R. Post, S. Griffith, J. Levitan, S. R. Manalis, M. A. Schmidt, and C. F. Quate, Appl. Phys. Lett. 76, 3316 (2000).

${ }^{2}$ O. Manzardo, H. P. Herzig, C. R. Marxer, and N. F. de Rooij, Opt. Lett. 24, 1705 (1999).

${ }^{3}$ M. Minoru Sasaki, X. Mi, and K. Hane, Appl. Phys. Lett. 75, 2008 (1999).

${ }^{4}$ H. L. Kung, S. R. Bhalotra, J. D. Mansell, D. A. B. Miller, and J. S. Harris, Jr., IEEE J. Sel. Top. Quantum Electron. 8, 98 (2002).

${ }^{5}$ H. Stiebig, H. Büchner, E. Bunte, V. Mandryka, D. Knipp, and G. Jäger, Appl. Phys. Lett. 83, 12 (2003).

${ }^{6}$ H. Stiebig, D. Knipp, S. R. Bhalotra, H. L. Kung, and D. A. B. Miller, Sens. Actuators, A 120, 110 (2005).

${ }^{7}$ W. Luft and Y. Tuso, Hydrogenated Amorphous Silicon Alloy Deposition Processes (Marcel Dekker, New York, 1993).

${ }^{8}$ O. Kluth, A. Löffl, S. Wieder, C. Beneking, L. Houben, B. Rech, H. Wagner, S. Waser, J. A. Selvan, and H. Keppner, Proc. IEEE 715 (1997). 